\title{
How herd best linear unbiased estimates affect the progress achievable from gains in additive and nonadditive genetic merit
}

\author{
F. L. Dunne, ${ }^{1,2}$ S. McParland, ${ }^{1}$ M. M. Kelleher, ${ }^{3}$ S. W. Walsh, ${ }^{2}$ and D. P. Berry ${ }^{1 *}$ \\ ${ }^{1}$ Teagasc, Animal and Grassland Research and Innovation Center, Moorepark, Fermoy, Co. Cork, Ireland P61 C996 \\ ${ }^{2}$ Waterford Institute of Technology, Cork Road, Waterford, Co. Waterford, Ireland X91 K0EK \\ ${ }^{3}$ Irish Cattle Breeding Federation, Bandon, Co. Cork, Ireland P72 X050
}

\section{ABSTRACT}

Sustainable dairy cow performance relies on coevolution in the development of breeding and management strategies. Tailoring breeding programs to herd performance metrics facilitates improved responses to breeding decisions. Although herd-level raw metrics on performance are useful, implicitly included within such statistics is the mean herd genetic merit. The objective of the present study was to quantify the expected response from selection decisions on additive and nonadditive merit by herd performance metrics independent of herd mean genetic merit. Performance traits considered in the present study were age at first calving, milk yield, calving to first service, number of services, calving interval, and survival. Herd-level best linear unbiased estimates (BLUE) for each performance trait were available on a maximum of 1,059 herds, stratified as best, average, and worst for each performance trait separately. The analyses performed included (1) the estimation of (co)variance for each trait in the 3 BLUE environments and (2) the regression of cow-level phenotypic performance on either the respective estimated breeding value (EBV) or the heterosis coefficient of the cow. A fundamental assumption of genetic evaluations is that 1 unit change in EBV equates to a 1 unit change in the respective phenotype; results from the present study, however, suggest that the realization of the change in phenotypic performance is largely dependent on the herd BLUE for that trait. Herds achieving more yield, on average, than expected from their mean genetic merit, had a $20 \%$ greater response to changes in EBV as well as $43 \%$ greater genetic standard deviation relative to herds within the worst BLUE for milk yield. Conversely, phenotypic performance in fertility traits (with the exception of calving to first service) tended to have a greater response to selection as well as a greater additive genetic standard deviation within

Received December 7, 2018.

Accepted February 12, 2019.

*Corresponding author: donagh.berry@teagasc.ie the respective worst herd BLUE environments; this is suggested to be due to animals performing under more challenging environments leading to larger achievable gains. The attempts to exploit nonadditive genetic effects such as heterosis are often the basis of promoting cross-breeding, yet the results from the present study suggest that improvements in phenotypic performance is largely dependent on the environment. The largest gains due to heterotic effects tended to be within the most stressful (i.e., worst) BLUE environment for all traits, thus suggesting the heterosis effects can be beneficial in mitigating against poorer environments.

Key words: best linear unbiased estimates, dairy, management, genotype by environment

\section{INTRODUCTION}

Genetic effects (i.e., additive and nonadditive) and environmental effects (i.e., permanent and temporary) as well as their interaction and, where relevant, their covariance, determine an animal's phenotypic performance (Visscher et al., 2008). Dairy cow breeding programs have focused almost exclusively on exploiting the additive genetic portion of the phenotype; crossbreeding strategies attempt to also exploit nonadditive genetic effects. Dairy cow genetic evaluations calculate the EBV of traits independent of measurable management effects through a process called BLUP.

The EBV, generally within the framework of an overall breeding objective, are used to rank animals as candidate parents of the next generation. Such ranking on EBV is undertaken irrespective of the environment/ management system their progeny are likely to be exposed to. Moreover, the expectation is that a 1 unit difference in EBV should equate to a 1 unit difference in performance (in the average environment). The existence of genotype by environment $(\mathbf{G} \times \mathbf{E})$ interactions contributes to a deviation from the expectation and such deviations have been documented in dairy cattle (Craig et al., 2018), beef cattle (Ferreira et al., 2015), and sheep (Pollott and Greeff, 2004). Previous studies, however, stratified herds based on performance, 
which implicitly encapsulates the total genetic merit of the contributing animals. In the process of genetic evaluations, both fixed effects solutions (best linear unbiased estimates; BLUE) and random effects solutions (BLUP) are generated, each being independent of the other. Therefore, arguably, a more logical strategy to quantify the extent of $\mathrm{G} \times \mathrm{E}$ interactions would be to compare the expected response to selection across herds divergent on BLUE. To our knowledge, there is a void in the scientific literature on such an approach. Furthermore, interest in dairying is intensifying in crossbreeding strategies as a means to introduce added benefit from the resulting heterosis; whereas the presence of heterosis by environment $(\mathbf{H} \times \mathbf{E})$ interactions has been cited in dairy cow populations (Bryant et al., 2007; Penasa et al., 2010; Kargo et al., 2012), there is a paucity of information on such interactions where the environments investigated are based on herd-level BLUE.

The objective of the present study was first to investigate the existence of $\mathrm{G} \times \mathrm{E}$ interactions across fertility, production, and survival traits by stratifying herds based on BLUE performance rather than phenotypic performances, thus excluding genetic effects. Second, the results from the present study aim to estimate the gains achievable when improving animal-level breeding values within 3 environments stratified on BLUE. Finally, the third objective of the present study was to identify the type of environment (e.g., superior versus poor) stratified on BLUE that expressed nonadditive genetic effects such as heterosis and estimate gains attainable within each environment from heterotic effects.

\section{MATERIALS AND METHODS}

Data used in the present study were obtained from the Irish Cattle Breeding Federation national database, Bandon, Co. Cork, Ireland (http://www.icbf.com).

\section{Quantifying BLUE}

The Irish national fertility genetic evaluation for dairy cows is a $23 \times 23$ multi-trait and multi-breed evaluation operated using the Mix99 software suite (MiX99 Development Team, 2015) in which each parity is treated as a separate trait. Traits evaluated in the multi-trait evaluation include age at first calving (AFC; i.e., the number of days from birth to first calving), milk yield ( $\mathrm{kg}$; based on a 305-d lactation yield) for parity 1 to 5 inclusive, calving to first service interval from parity 1 to 3 inclusive (CFS; i.e., the number of days from calving to the cow receiving her first service), number of services (NS) from parity 1 to 3 inclusive, and calving interval from parity 1 to 5 inclusive (CIV; i.e., the number of days between subsequent calving events). Survival is evaluated as a binary trait based on parity 1 to 5 (inclusive) and is recorded as 1 for lactation $x-1$ where a recorded calving date existed for lactation $x$, otherwise as 0 (Berry et al., 2013). Fixed effects included in all models are age at calving (unless age at first calving is the dependent variable), heterosis, and recombination loss coefficients; heterosis coefficients were split into separate Holstein $\times$ Friesian $(\mathbf{H O} \times \mathbf{F R})$, Holstein $\times$ Jersey $(\mathbf{H O} \times \mathbf{J E})$, Holstein $\times$ Montbelliarde $(\mathbf{H O} \times \mathbf{M O})$, Holstein $\times$ Meuse Rhine Yssel $(\mathbf{H O} \times \mathbf{M Y})$, and Shorthorn $\times$ HolsteinFriesian $(\mathbf{S R} \times \mathbf{H F})$ coefficients. Contemporary group of herd-year-season of calving or birth (AFC) is also included as a fixed effect. Within the genetic evaluation, contemporary group (HYS) effects are defined using methodology outlined in Berry et al. (2013) formed based on algorithms proposed by Schmitz et al. (1991) and Crump et al. (1997); animals within the same herd are grouped based on their calving dates or birth dates (in the case of AFC). The fixed effects and random effects solutions of 3,445,158 dairy cows were obtained from the August 2017 national genetic evaluation for use in the present study.

\section{Data Editing for Herd-Level BLUE}

Contemporary group BLUE for the years 2012 to 2014 (inclusive) and individual animal EBV were extracted from the national genetic evaluation, for actively milk recording, spring-calving herds. Spring-calving herds, which predominate in Ireland (Berry et al., 2013), were defined as herds calving at least $80 \%$ of their cows between January and June (inclusive). Data from 4,129 herds were available for analysis. Parity-level BLUE solutions for each trait were initially base-adjusted to a common base population; the base population consisted of 152 milk recording herds that had a minimum of 3 records for AFC as well as a minimum of 4 records available for parity 1 to 3 inclusive for NS, CFS, and parity 1 to 5 for CIV, survival, and milk yield between the years 2012 to 2014 inclusive. A single collapsed herd-year BLUE for each trait was calculated as the average of each HYS estimate in the herd, weighted by the number of animal records within each HYS (Dunne et al., 2018). Subsequently, the single collapsed herdyear BLUE was calculated to be relative to the mean of the sample population.

Herds were removed from the study if they did not meet the following criteria during the study period: (1) herds were required to have a BLUE available for all 6 investigated traits; 2,185 herds remained; (2) a minimum of 5 records within each contemporary group were required for at least 2 of 3 parities for CFS or NS, and 
4 of 5 parities for CIV, SU, and milk yield (Dunne et al., 2018); 1,911 herds remained; and (3) herds were required to have phenotypic data available on a minimum of 45 animals and 30 calving events per year; where AFC was the trait analyzed, herds were required to have at least 10 heifers calving for the first time each year. The final data set consisted of 1,764 herds with 124,352 animals available for the AFC analysis and 216,270 animals available for the analysis of the other performance traits.

\section{Stratifying Herd BLUE for Validation Population}

A single weighted herd BLUE was calculated by averaging the respective herd-year BLUE across the $3 \mathrm{yr}$ of data. Herds were ranked on each trait separately. After stratifying the herd-BLUE into 5 strata, only the first, third, and fifth stratum which represented the top, average, and bottom performing 20 percentiles, respectively, were retained. As herd BLUE were stratified based on each trait individually, herds could rank differently for each trait. The final data set analyzed for AFC, CFS, NS, CIV, survival, and milk yield contained 1,059 herds (71,721 animals), 1,058 herds (125,736 animals), 1,058 herds (130,607 animals), 1,059 herds (125,035 animals), 1,058 herds (125,934 animals), and 1,058 herds (131,695 animals), respectively.

The phenotypic records of animals within the retained herds were masked and the genetic evaluation rerun with the remaining $3,234,629$ animals; this was done to generate EBV for animals within the retained herds without their own performance data contributing to their EBV. The EBV generated from the subsequent genetic evaluation were then base-adjusted to a base population of 414 Holstein-Friesian AI sires that were born between 2000 and 2005, inclusive.

\section{Statistical Analyses}

Two separate approaches were used to quantify the extent of $\mathrm{G} \times \mathrm{E}$ interactions. First (co)variance component analyses were undertaken using a series of within-trait bivariate sire linear mixed models in ASReml (Gilmour et al., 2009) where the phenotypic performance in each herd BLUE stratum was considered a different trait. The linear mixed model applied to each trait was

$$
\begin{aligned}
& Y_{i j k l m n x}=H Y S_{j}+\text { Parity }_{k}+\text { Heterosis }_{l}+\text { Recombination }_{m} \\
& +\sum_{n=1}^{2} a g e^{n}+s_{x}+e_{i j k l m n o}
\end{aligned}
$$

in which $Y_{i j k l m n x}=$ the observed phenotypic performance for AFC, CFS, NS, CIV, survival, or milk yield of ani- mal $i$ in a given BLUE stratum; $H Y S_{j}=$ the fixed effect of contemporary group; Parity ${ }_{k}=$ the fixed effect of the $k$ th parity (i.e., $1,2,3,4,5$ ) of the animal $i$ (not included in AFC); $a g e^{n}=$ the covariate of age (linear and quadratic) of animal $i$ (not included in AFC); Heterosis $=$ the covariate representing the total heterosis coefficient $l$ of animal $i$; Recombination ${ }_{m}=$ the covariate representing the recombination coefficient $m$ of animal $i ; s_{x}=$ the random effect of the sire additive genetic component of animal $i$, where $s \sim N\left(\mathbf{0}, \mathbf{A} \sigma_{s}^{2}\right)$ with $\sigma_{s}^{2}$ representing the additive genetic variance of the sire and A the additive genetic relationship matrix among sires; $e_{i j k l m n o}=$ random residual effect, where $e \sim N\left(\mathbf{0}, \mathbf{I} \sigma_{e}^{2}\right)$ with $\sigma_{e}^{2}$ representing the residual variance with a separate residual variance fitted for each BLUE stratum and I representing the identity matrix. While a separate residual variance was estimated in each environment, no residual covariance was assumed between records in the different environments. Therefore, the detailed (co)variance structure for a 2-trait bivariate analysis between trait $x$ and $y$ was

$$
\begin{gathered}
{\left[\begin{array}{l}
s_{x} \\
s_{y}
\end{array}\right]=N\left(\left[\begin{array}{l}
0 \\
0
\end{array}\right],\left[\begin{array}{cc}
\mathbf{A}_{x x} \sigma_{s_{x}}^{2} & \mathbf{A}_{x y} \sigma_{s_{x y}} \\
\mathbf{A}_{y x} \sigma_{s_{y x}} & \mathbf{A}_{y y} \sigma_{s_{y}}^{2}
\end{array}\right]\right),} \\
{\left[\begin{array}{l}
e_{x} \\
e_{y}
\end{array}\right]=N\left(\left[\begin{array}{l}
0 \\
0
\end{array}\right],\left[\begin{array}{cc}
\mathbf{I} \sigma_{e_{x}}^{2} & 0 \\
0 & \mathbf{I} \sigma_{e_{y}}^{2}
\end{array}\right]\right),}
\end{gathered}
$$

where $\mathbf{A}$ is the numerator relationship matrix; $\sigma_{s_{x}}^{2}, \sigma_{s_{y}}^{2}$, $\sigma_{s_{x y}}$, and $\sigma_{s_{y x}}$ represent the sire genetic (co)variance parameters of the sires in BLUE stratum $x$ and $y$, respectively; and $\sigma_{e_{x}}^{2}$ and $\sigma_{e_{y}}^{2}$ are the residual variance in BLUE stratum $x$ and $y$, respectively. The estimated genetic variance in each stratum was obtained as 4 times the estimated sire variance. Whether the variance components in each herd BLUE stratum differed from each other was determined using a likelihood ratio test of nested models comparing an unconstrained model to a model where the genetic variance in each stratum of the bivariate analysis was constrained to be equal. Heritability estimates were calculated using ASReml (Gilmour et al., 2009) by dividing the genetic variance (i.e., 4 times the sire genetic variance) by the phenotypic variance (i.e., the sum of the residual and genetic variance).

In the second analysis strategy, multivariate linear regression models were used to regress each phenotypic performance trait on its respective EBV in each of the 3 BLUE strata for all performance traits using PROC 
MIXED in SAS 9.4 software (SAS Institute Inc., Cary, NC). The linear regression model fitted for all traits was

$$
\begin{aligned}
& Y_{i j k l m n o p}=H Y S_{j}+\text { Parity }_{k}+B L U E_{l} \mid \sum_{m=1}^{6} \text { Heterosis }_{m} \\
& +\sum_{n=1}^{2} a g e^{n}+B L U E_{l}\left|E B V_{o}+B L U E_{l}\right| \text { recombintation }_{p} \\
& +e_{i j k l m n o p},
\end{aligned}
$$

where $Y_{i j k l m n o p}=$ the observed phenotypic performance for AFC, CFS, NS, CIV, survival, or milk yield of animal $i$ in BLUE stratum $l ; H Y S_{j}=$ the fixed contemporary group effect; Parity $_{k}=$ the fixed effect of the $k$ th parity (i.e., $1,2,3,4,5$ ) of animal $i$ (not included when the dependent variable was $\mathrm{AFC}) ; B L U E_{l}=$ the fixed effects of $l$ th BLUE stratum $(\mathrm{n}=3)$; Heterosis ${ }_{m}$ $=$ the covariate representing the heterosis coefficient $m$ of animal $i$ for the breed crosses $\mathrm{HO} \times \mathrm{FR}, \mathrm{HO}$ $\times \mathrm{JE}, \mathrm{HO} \times \mathrm{MO}, \mathrm{HO} \times \mathrm{MY}, \mathrm{SR} \times \mathrm{HF}$, and other; $a g e^{n}=$ the covariate of age (linear and quadratic) of animal $i$ (not included when the dependent variable was $\mathrm{AFC}) ; E B V_{o}=$ the estimated breeding value $o$ for animal $i$; recombination , $_{p}=$ the covariate representing the total recombination coefficient value $p$ of animal $i$; and $e_{i j k l m n o p}=$ the residual error effect.

\section{RESULTS}

Mean phenotypic performance for all traits improved as the respective herd mean BLUE stratum improved (Table 1). Similarly, the mean EBV for 305-d milk yield improved as herd stratum for mean milk BLUE improved, differing by $163 \mathrm{~kg}$ between the best and worst strata. Herds with the shortest EBV for AFC and CIV were within the respective average stratum, the longest (i.e., worst) $\mathrm{EBV}$ for $\mathrm{AFC}$ was within the best stratum, whereas the longest (i.e., worst) EBV for CIV was within the worst stratum (Table 1). The mean EBV for CFS was longest (i.e., worst) in the best stratum for CFS, whereas no differences existed between the average and worst strata for CFS $(P=0.535)$. The mean EBV for NS differed $(P<0.05)$ between the 3 BLUE strata for NS, whereas the EBV for survival only differed $(P<0.001)$ between the best and worst herd BLUE strata for survival (Table 1).

\section{$G \times E$ Interactions}

The largest genetic standard deviation for AFC was within the worst stratum for AFC, being 2.5 times greater $(P=0.001)$ than the genetic standard deviation of AFC in the average stratum. As the BLUE for milk yield improved, the genetic standard deviation of milk yield increased by a factor of $1.43(P<0.001)$ between the worst and best strata for milk yield (Table 2). The genetic standard deviation for NS was 3.25 times larger $(P<0.001)$ in the worst NS BLUE stratum than in the best stratum for NS BLUE. No differences existed in the genetic standard deviation of survival between the 3 BLUE strata for survival (Table 2). With the exception of AFC, the genetic correlations between strata for the remaining traits were all $\geq 0.827$, thus indicating that re-ranking effects are absent across the different BLUE strata (Table 2). Genetic correlations

Table 1. Mean (SD in parentheses) phenotypic performance, herd best linear unbiased estimate (BLUE), and EBV of animals within herds stratified as best (top 20\%), average (middle 20\%), and worst (bottom 20\%) for the BLUE of the respective performance trait

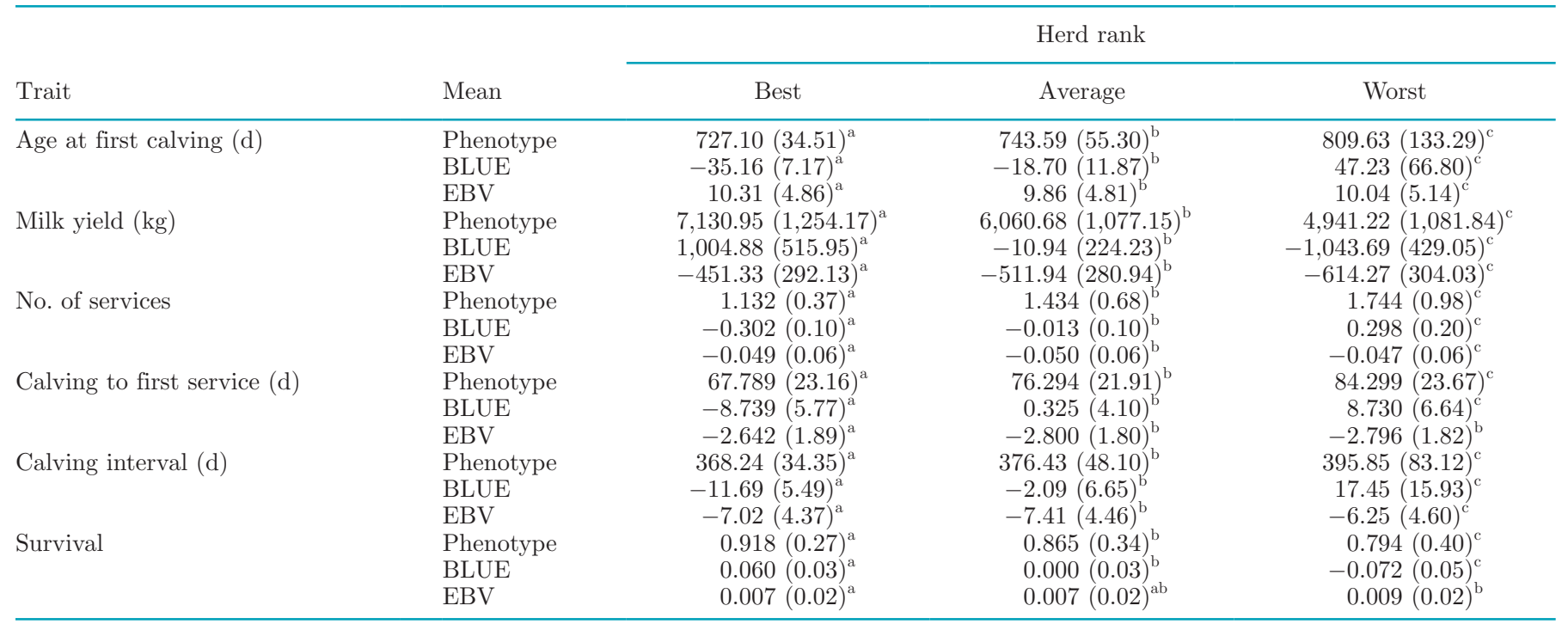

\footnotetext{
${ }^{\mathrm{a}-\mathrm{c}}$ Values within a row with different superscripts differ $(P<0.05)$.
} 
Table 2. Additive genetic SD for each performance trait within herds stratified as best (top 20\%), average (middle 20\%), and worst (bottom $20 \%$ ) based on the best linear unbiased estimates (BLUE) for the respective performance trait as well as the genetic correlations (SE in parentheses) between the strata

\begin{tabular}{|c|c|c|c|c|c|c|}
\hline BLUE trait & \multicolumn{3}{|c|}{ Genetic SD } & \multicolumn{3}{|c|}{ Correlation } \\
\hline Age at first calving & $14.05^{\mathrm{ab}}$ & $12.64^{\mathrm{a}}$ & $31.67^{\mathrm{b}}$ & $0.275(0.163)$ & $-0.504(0.132)$ & $0.514(0.167)$ \\
\hline Calving to first service & 2.39 & 2.23 & 2.83 & $0.869(0.465)$ & $0.983(0.142)$ & $0.827(0.161)$ \\
\hline No. of services & $0.04^{\mathrm{a}}$ & $0.09^{\mathrm{a}}$ & $0.13^{\mathrm{b}}$ & $0.912(0.226)$ & $0.960(0.216)$ & $0.898(0.137)$ \\
\hline Calving interval & 3.07 & 5.08 & 6.81 & $0.932(0.276)$ & $0.961(0.206)$ & $0.905(0.291)$ \\
\hline
\end{tabular}

for AFC ranged from -0.504 (between the best and worst stratum) to 0.514 (between the average and worst stratum; Table 2).

A tendency was observed for the largest residual standard deviation to be within the worst BLUE environment and the smallest residual variance to be within the best environment for all traits with the exception of milk yield and CFS (Table 3). The heritability of milk yield increased as the herd environment BLUE improved ranging from 33 to $50 \%$ (Table 3 ). With the exception of CIV, the heritability estimates for the remaining fertility traits tended to increase as the respective environment worsened, ranging from 0.01 to 0.02 (Table 3).

\section{EBV Regression Analyses}

The regression of phenotypic survival on the EBV for survival did not differ by herd BLUE strata for either milk yield, CIV, or CFS (Table 4). Similarly, the relationship between CFS phenotype and the EBV for CFS did not differ by BLUE strata for either CFS or survival (Table 4). The regression coefficient of phenotypic AFC on the EBV for AFC within the worst BLUE stratum for AFC was 4 times that in the best BLUE stratum for AFC.
The response in phenotypic milk yield per kilogram increase in milk EBV in the best milk BLUE stratum was $20 \%$ greater than observed in the worst milk BLUE stratum $(P<0.001)$. The regression of phenotypic CIV on the EBV for CIV in the best milk yield BLUE stratum was 1.63 times $(P<0.001)$ that of the regression coefficient in the worst milk BLUE stratum; the corresponding factor for NS was $2.52(P<0.001)$. The improvement in phenotypic CFS regressed on the EBV for CFS within the worst milk yield BLUE stratum was $1.69(P<0.01)$ times greater than that in the average stratum for milk yield (Table 4 ).

As the BLUE for CIV worsened, the regression coefficient of phenotypic CIV on the EBV for CIV became greater, differing by a factor of 1.63 (between the best and average strata; $P<0.05$ ) to 2.63 (between the average and worst strata; $P<0.001)$ compared with the more superior BLUE (Table 4). Phenotypic milk yield, CFS, and NS regressed on the respective EBV ranged between 1.27 (milk yield; $P<0.001$ ) to 1.93 $(\mathrm{NS} ; P<0.001)$ times greater within the worst stratum BLUE for CIV than the best BLUE stratum for CIV (Table 4).

Although no differences existed between the regression coefficients of phenotypic CFS on the EBV for CFS within the 3 BLUE strata for CFS, the regression

Table 3. Residual SD for each performance trait within herds stratified as best (top 20\%), average (middle 20\%), and worst (bottom 20\%) based on the best linear unbiased estimates (BLUE) for the respective performance trait as well as the heritability estimates of each trait within the best, average, and worst strata

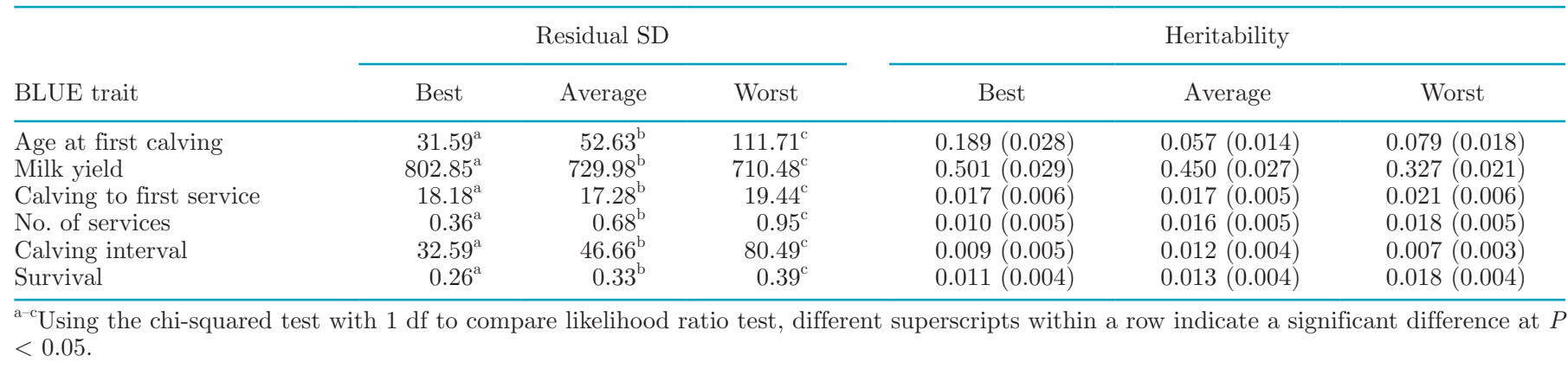


Table 4. Regression coefficients (SE within parentheses) of phenotypic values of each trait regressed on EBV for each respective trait across the best (top 20\%), average (middle 20\%), and worst (bottom 20\%) strata of best linear unbiased estimates (BLUE)

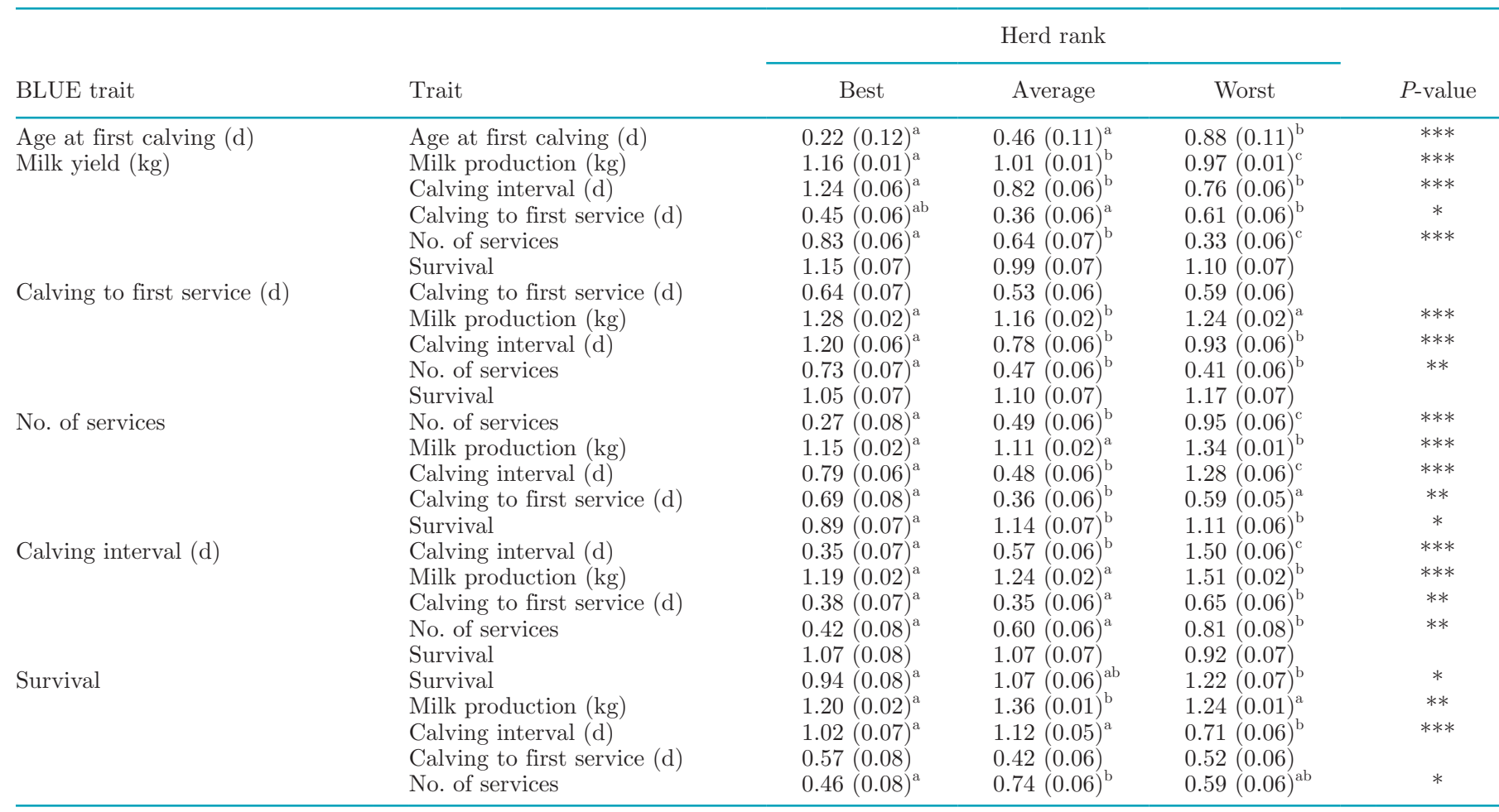

${ }^{\mathrm{a}-\mathrm{c}}$ Values within a row with different superscripts differ $(P<0.05)$.

${ }^{*} P<0.05,{ }^{* *} P<0.01,{ }^{* * *} P<0.001$.

coefficient of milk yield on the EBV for milk yield was 1.10 times greater $(P<0.001)$ within the best BLUE stratum for CFS compared with the average BLUE stratum for CFS (Table 4). Within the best stratum for CFS, the regression coefficients of CIV and NS on the respective EBV were $1.29(\mathrm{CIV} ; P=0.002)$ and $1.78(\mathrm{NS} ; P=0.001)$ times greater than that of the respective regression coefficients within the worst BLUE stratum for CFS (Table 4).

Phenotypic NS regressed on the EBV for NS was 3.52 times greater $(P<0.001)$ within the worst BLUE stratum for NS relative to the best stratum for NS, whereas the regression coefficient of phenotypic milk yield and CIV regressed on the respective EBV was $1.21(P<0.001)$ and $2.67(P<0.001)$ times greater within the worst BLUE stratum for NS than the average stratum for NS (Table 4). Within the best stratum for NS, the regression coefficient of CFS on the EBV for CFS was $1.92(P<0.001)$ times greater than in the average stratum for NS. Conversely, within the average stratum for NS, the regression coefficient of survival on the EBV for survival was $1.28(P=0.01)$ times greater than that in the best BLUE stratum for NS (Table 4).

The response in phenotypic survival per unit increase in the EBV for survival was 1.30 times $(P=0.006)$ greater within the worst stratum for survival relative to the best BLUE stratum. Within the average stratum for survival, the regression coefficients of phenotypic milk yield and NS were $1.13(P<0.001)$ and $1.61(P$ $=0.004)$ times greater, respectively, than in the best stratum for survival. Although the regression coefficient of CIV regressed on the EBV for CIV was 1.58 times greater $(P<0.001)$ within the average stratum for survival in comparison to the worst stratum, no differences existed between the best and average stratum for survival (Table 4).

\section{Heterosis Coefficient Regression Analysis Within BLUE Stratum}

The regression of phenotypic $\mathrm{AFC}$ on the $\mathrm{HO} \times \mathrm{JE}$ heterosis coefficient was 3.65 times greater $(P=0.04)$ within the worst BLUE stratum for AFC than within the best BLUE stratum for AFC (Table 5). Phenotypic milk yield regressed on the $\mathrm{HO} \times \mathrm{JE}$ heterosis coefficient was greatest in the average milk BLUE stratum, being 4.37 times greater than in the best milk BLUE stratum $(P<0.001)$ and 1.40 times greater $(P<0.05)$ than in the worst milk BLUE stratum. The regression coefficient of phenotypic milk yield on the $\mathrm{HO} \times \mathrm{FR}$ heterosis coefficient was 1.86 times greater $(P<0.001)$ 
Table 5. Linear regression coefficients of phenotypic performance for each trait on Holstein $\times$ Friesian heterosis coefficient $(\mathrm{HO} \times \mathrm{FR})$, Holstein $\times$ Jersey heterosis coefficient $(\mathrm{HO} \times \mathrm{JE})$, and recombination coefficient within each herd best linear unbiased estimate (BLUE) stratum including the significance of the interaction

\begin{tabular}{|c|c|c|c|c|}
\hline BLUE trait & \multicolumn{3}{|c|}{ Herd rank } & $P$-value \\
\hline \multicolumn{5}{|c|}{ Age at first calving (d) } \\
\hline $\mathrm{HO} \times \mathrm{JE}$ & $-3.98(2.58)^{\mathrm{a}}$ & $-7.12(3.13)^{\mathrm{ab}}$ & $-14.53(4.34)^{\mathrm{b}}$ & * \\
\hline Recombination & $-10.70(3.66)^{\mathrm{a}}$ & $-11.93(3.6)^{a}$ & $-47.83(4.28)^{\mathrm{b}}$ & $* * *$ \\
\hline \multicolumn{5}{|l|}{ Milk yield (kg) } \\
\hline \multicolumn{5}{|l|}{ Survival } \\
\hline $\mathrm{HO} \times \mathrm{FR}$ & $0.01(0.01)^{\mathrm{a}}$ & $0.02(0.01)^{\mathrm{a}}$ & $0.04(0.01)^{\mathrm{b}}$ & $* * *$ \\
\hline $\mathrm{HO} \times \mathrm{JE}$ & $0.03(0.01)$ & $0.04(0.01)$ & $0.04(0.01)$ & \\
\hline Recombination & $-0.04(0.01)^{\mathrm{a}}$ & $0.00(0.01)^{b}$ & $-0.02(0.01)^{\mathrm{b}}$ & ** \\
\hline \multicolumn{5}{|l|}{ No. of services } \\
\hline $\mathrm{HO} \times \mathrm{FR}$ & $-0.03(0.02)$ & $-0.04(0.02)$ & $-0.09(0.02)$ & \\
\hline \multicolumn{5}{|c|}{ Calving to first service interval (d) } \\
\hline Recombination & $-1.88(1.03)$ & $-2.10(0.82)$ & $-2.79(0.77)$ & \\
\hline \multicolumn{5}{|c|}{ Calving interval (d) } \\
\hline $\mathrm{HO} \times \mathrm{FR}$ & $-0.82(1.26)^{\mathrm{a}}$ & $-2.64(1.09)^{\mathrm{a}}$ & $-9.77(1.22)^{\mathrm{b}}$ & * \\
\hline $\mathrm{HO} \times \mathrm{JE}$ & $-3.26(1.91)^{\mathrm{a}}$ & $-3.31(1.41)^{\mathrm{a}}$ & $-6.04(1.42)^{\mathrm{a}}$ & $*$ \\
\hline Recombination & $1.07(2.30)$ & $-0.47(1.82)$ & $-6.42(1.95)$ & \\
\hline
\end{tabular}

${ }^{\mathrm{a}-\mathrm{c}}$ Values within a row with different superscripts differ $(P<0.05)$.

${ }^{*} P<0.05,{ }^{* *} P<0.01,{ }^{* * *} P<0.001$.

in the worst milk BLUE stratum relative to the best stratum (Table 5). The regression of phenotypic CFS, $\mathrm{NS}$, and CIV on the HO $\times \mathrm{JE}$ heterosis coefficient was $1.86(\mathrm{CIV})$ to $2.40(\mathrm{NS})$ times greater within the respective worst BLUE stratum than the best BLUE stratum. Within the worst BLUE stratum for CIV, the regression coefficient of phenotypic CIV on the $\mathrm{HO}$ $\times$ FR heterosis coefficient was almost 12 times $(P<$ $0.001)$ that in the best BLUE stratum for CIV. Phenotypic survival regressed on the $\mathrm{HO} \times \mathrm{FR}$ heterosis coefficient was 4 times greater $(P<0.001)$ within the worst stratum for survival in comparison to the best BLUE stratum (Table 5). The regression coefficients of phenotypic performance on each of the heterosis coefficients stemming from the breed combinations $\mathrm{HO} \times$ $\mathrm{MO}, \mathrm{HO} \times \mathrm{MY}$, and $\mathrm{SR} \times \mathrm{HF}$ are in Supplemental Table S1 (https://doi.org/10.3168/jds.2018-16119). Although a similar trend existed for all traits (with the exception of CIV), the associated standard errors were large owing to the small representation of these breed crosses in the data set.

\section{Recombination Coefficient Regression Analysis Within BLUE Stratum}

The regression of phenotypic AFC on the recombination coefficient was 4.47 times greater $(P<0.001)$ in the worst stratum than in the best stratum for AFC (Table 5). Phenotypic milk yield regressed on the recombination coefficient was 4.56 times greater $(P<$ 0.001 ) in the best BLUE stratum for milk yield than the respective average stratum (Table 5). The regression coefficient of phenotypic NS on the recombination coefficient was 2.4 times greater $(P<0.001)$ in the opposite direction within the best BLUE stratum than in the worst BLUE stratum for NS (Table 5). Phenotypic survival regressed on the recombination coefficient ranged from 0.00 (in the average stratum) to -0.043 (in the best stratum; Table 5).

\section{DISCUSSION}

Breeding programs tailored to the requirements of individual dairy producers are increasingly sought after. The expected performance of the progeny from a given mating is traditionally predicted from the EBV of the progeny converted to a deviation from the mean phenotypic performance of the base animal; such an approach assumes that a 1 unit difference in EBV equates to a 1 unit phenotypic difference for that trait. Similarly, if the progeny is crossbred, then a predetermined heterosis effect is added to the respective predicted phenotypic performance to generate an estimate of expected per- 
formance (Kelleher et al., 2016). Results from the present study reveal that such simple mathematics are not always valid, and not only does the association between EBV and phenotype differ by environment, but also the association between the heterosis coefficient and phenotype differs by environment. The former phenomenon, commonly known as $\mathrm{G} \times \mathrm{E}$, has been well publicized (Fikse et al., 2003; Haile-Mariam et al., 2008; Craig et al., 2018), whereas the latter, known as $\mathrm{H} \times \mathrm{E}$ interaction, is less well documented in dairy cows (Penasa et al., 2010; Kargo et al., 2012). To our knowledge no study has attempted to quantify the extent of either $\mathrm{G} \times \mathrm{E}$ or $\mathrm{H} \times \mathrm{E}$ where the environment (i.e., $\mathrm{E}$ ) was represented by a BLUE from a genetic evaluation.

Dunne et al. (2018), using a subset of the data used in the present study, proposed the use of herd-level BLUE to categorize dairy herds based on performance independent of genetic effects and systematic environmental effects. The motivation for using the BLUE was to provide informed decision support systems especially for the identification of herds performing below their genetic potential and, by using the fixed effects solutions from the underlying statistical model, attempt to explain some of the apparent inconsistencies (Dunne et al., 2018). Once diagnosed, the next step in a decision support system is to provide solutions to help improve herd performance. Although management strategies such as improved animal nutrition, vaccination, or greater attention to reproductive performance could help bridge some of the gap in the difference between expectation and realization, breeding strategies could also form part of the solution. Results from the present study clearly showed that the realization of breeding decisions is associated with the environment the animal is/was exposed to, and therefore incorporating such information in a mating advice tool may be beneficial in the prediction of realistic offspring performance. Being able to derive expected performance of offspring for alternative scenarios may also be useful in the evaluation of alternative breeding strategies such as, for example, evaluating the benefit of a cross-breeding program.

\section{Genetic Variability Differs by Environment}

The observation of a larger genetic standard deviation for milk yield in the high milk BLUE environment (1.43 times than that of the worst milk BLUE; Table 2) was consistent with the greater regression coefficient of phenotypic milk yield on the milk yield EBV (1.2 times greater) in the high BLUE than in the worst milk BLUE (Table 4); this indicates that the mean response to selection for milk yield is greater in the best BLUE for milk yield. The converse was true, however, for the remaining traits representing fertility (with the excep- tion of CFS), AFC, and survival, in that the regression coefficient of the phenotypic performance on the respective EBV ranged from 1.30 (survival) to 4.28 (CIV; d) times larger in the respective worst BLUE environment relative to the best BLUE environment. Similarly, the genetic standard deviation of each trait (except milk yield) was 1.18 (CFS; d) to 3.25 (NS; serves) times larger in the respective worst BLUE environment relative to the respective best BLUE environment. Craig et al. (2018) reported a similar range (1.2 to 3.6 times greater) when estimating the genetic standard deviations of fertility traits (i.e., calving rate within the first $42 \mathrm{~d}$ of the calving season, calving season day, and the percentage mated in the first $21 \mathrm{~d}$ of the calving season) within low-fertility herd environments relative to highfertility environments. Low genetic standard deviations for survival within the best respective stratum relative to the worst stratum may be an indication of more stringent voluntary culling decisions and may explain why the mean EBV for survival was greatest in the worst stratum for survival.

The differential in genetic standard deviations and the regression coefficients of the phenotypic performance on the respective EBV by environment is not novel and has been reported elsewhere in dairy cattle (Craig et al., 2018), beef cattle (Ferreira et al., 2015), and sheep (Pollott and Greeff, 2004). The novelty here, however, was that no study to date has stratified environments based on BLUE and therefore there was an implicit link between the herd stratum for the performance trait and genetic merit because the mean genetic merit of the herds would have contributed to their eventual phenotypic performance; this is especially true for highly heritable traits such as milk yield. Dunne et al. (2018) analyzed BLUE in the form of HYS effects and reported that from the sum of the regression coefficient of BLUE and BLUP fitted through $10 \mathrm{yr}$ of data, $69 \%$ of the improvement in phenotypic milk yield was as a result of BLUE effects. The greater genetic standard deviation of milk yield in the best BLUE milk yield environment therefore clearly suggests that it is largely a function of the superior management employed in these environments, which in Ireland could include superior grassland management but also could be due to increased concentrate input. From a controlled experimental study on grazing dairy cows, Coleman et al. (2010) documented a greater response in milk solids to feed intake in higher genetic merit animals (based on a total merit index) from either a greater inclusion of concentrate or grass allowance in the diet.

Greater regression coefficients of fertility phenotypic performances on the respective EBV within the respective worst BLUE stratum are indicative of a greater response to selection for fertility traits in such envi- 
ronments. Yet, unlike milk yield, where poor response to selection is reflective of sub-optimum management, the poor response to selection within the best BLUE fertility trait stratum is reflective of superior management under optimal environmental conditions leading to animals approaching their biological limit or the limit of the production system (i.e., calving intervals of less than 365 are generally not sought at a herd level). Management practice obviously have an effect on reproductive performance as evidenced by a mean phenotypic difference of $27.61 \mathrm{~d}$ between the best and worse CIV BLUE despite a mean difference of less than $1 \mathrm{~d}$ in EBV for CIV (Table 1).

With the exception of AFC, genetic correlations for the same trait in different strata were in the range of 0.827 (between average and worst strata for CFS BLUE; SE = 0.161) to 0.983 (between best and worst strata for CFS BLUE; SE $=0.142$; Table 2). Mulder et al. (2006) suggested that only genetic correlations between environments of $<0.61$ justified consideration of a separate breeding program to optimize genetic gain in the different environments. Although the correlations between BLUE strata for AFC were less than $0.61, \mathrm{AFC}$ is a trait that may reflect largely upon the breeding decisions of the manager and not necessarily the animal's genetic capability; the mating of heifers may be suspended to have the animals calving at a particular time of the year, especially in seasonal calving programs (Berry et al., 2013).

\section{Benefits of Heterosis Differ by Environment}

Heterosis is a nonadditive genetic phenomenon whereby the performance of crossbred progeny, on average, surpasses the mid-parent mean (Simm, 1998). Interest in crossbreeding strategies is increasing in dairy cow production systems as a means to rapidly improve performance, especially for traits associated with viability and fertility (McAllister, 2002; Wall et al., 2005; Sørensen et al., 2008). In some populations, however, the additive genetic merit of one dairy breed may actually be substantially inferior to that of the best available dairy breed. A decision is therefore required as to whether the direct progeny performance would be expected to be superior if crossbreeding was embarked upon (taking cognizance of expected heterosis) versus if straight-breeding with the genetically superior breed was undertaken. An estimate of the mean heterosis effect for different breed combination can be derived from published studies (Dezetter et al., 2015; Coffey et al., 2016) or simply as the fixed effects solutions from routine genetic evaluations.

Results from the present study clearly demonstrate that the benefit of heterosis differs by environment, which is consistent with reports elsewhere on production traits in dairy cows (Penasa et al., 2010; Kargo et al., 2012); however, there is a lack of $\mathrm{H} \times \mathrm{E}$ studies for fertility traits. Based on the results from the present study, (1) genetic evaluations should take cognizance of this $\mathrm{H} \times \mathrm{E}$ interaction, and (2) mating-based decision support systems need to apply the correct heterosis effects, based on herd BLUE, when comparing the mating of parents of the same or different breeds.

Animals within the best BLUE herds for production, fertility, and survival tended to experience the least benefit from heterosis, suggesting that heterotic effects are expressed more as the environment becomes increasingly stressful. The extent of heterosis expression, however, seems to be dependent on the breed composition of the $\mathrm{F}_{1}$ crosses when analyzing production traits. Based on Friesian crosses, Penasa et al. (2010) reported a similar trend to the present study whereby the greatest heterosis benefit was within the worst environment for milk yield. Nonetheless, the greatest benefit in milk yield from heterosis when Jersey formed a component of the cross in the present study was within the average herd BLUE for milk yield, which is consistent with Bryant et al. (2007) and Kargo et al. (2012). The heterosis coefficients of the fertility and survival traits were consistently larger in the respective worst BLUE strata (i.e., more stressful environment) relative to the best BLUE strata. This suggests that not only can performance (and profit) be increased in herds with poor BLUE for fertility and survivability with the exploitation of heterosis effects, but such effects may also promote robustness and resilience among animals within challenging environments. Within the Irish national economic breeding index, calving interval has a negative economic value of $€ 12.59$ per day (Irish Cattle Breeding Federation, 2017). Therefore, the economic benefit of heterosis in an $\mathrm{F}_{1} \mathrm{HO} \times \mathrm{FR}$ cross is expected, on average, to be $€ 123$ in herds within the poorest BLUE for calving interval but only $€ 10.32$ in the herds that are best for calving interval.

\section{CONCLUSIONS}

Phenotypic performance is inherently influenced by both the environment and genetic effects as well as how both effects interact with each other. Thus, predicting the additive genetic merit of a progeny from a given mating is suggested to be too simplistic and does not take cognizance of the environmental factors that are likely to influence the realization of the genetic merit. By stratifying herds on BLUE for each trait, the phenotypic response to changes in EBV differed for milk yield, CIV, NS, and AFC between at least 2 of the 3 environments investigated. Additive genetic variance 
for milk yield, AFC, and NS was greatest within the environment that had the largest scope for improvement (e.g., the best herd BLUE environment for milk yield and the worst herd BLUE environment for AFC and NS). Hence, accounting for the heterogeneity of variances in the genetic evaluation process is important. With the exception of the $\mathrm{HO} \times \mathrm{JE}$ heterosis for milk yield, the benefits of nonadditive heterotic effects were greatest in the most stressful environment for each respective trait. Ultimately, the results from the present study reiterate that heterosis is an effect that promotes robustness and resilience and is emphasized when conditions deteriorate. Clear benefits from incorporating BLUE into a breeding-specific decision support tool can facilitate more precise breeding decisions specific to individual herds.

\section{ACKNOWLEDGMENTS}

This publication emanated from research supported in part by a research grant from Science Foundation Ireland (Dublin, Ireland) and the Department of Agriculture, Food and Marine on behalf of the Government of Ireland (Dublin, Ireland) under the Grant 16/RC/3835 (VistaMilk) as well as funding from the Irish Department of Agriculture, Food and the Marine STIMULUS research grant MultiRepro (Dublin, Ireland).

\section{REFERENCES}

Berry, D. P., J. Kearney, K. Twomey, and R. Evans. 2013. Genetics of reproductive performance in seasonal calving dairy cattle production systems. Ir. J. Agric. Food Res. 52:1-16.

Bryant, J., N. López-Villalobos, J. Pryce, C. Holmes, D. Johnson, and D. Garrick. 2007. Short Communication: Effect of environment on the expression of breed and heterosis effects for production traits. J. Dairy Sci. 90:1548-1553. https://doi.org/10.3168/jds.S0022 -0302(07)71640-5.

Coffey, E., B. Horan, R. Evans, and D. Berry. 2016. Milk production and fertility performance of Holstein, Friesian, and Jersey purebred cows and their respective crosses in seasonal-calving commercial farms. J. Dairy Sci. 99:5681-5689. https://doi.org/10.3168/ jds.2015-10530.

Coleman, J., K. Pierce, D. Berry, A. Brennan, and B. Horan. 2010. Increasing milk solids production across lactation through genetic selection and intensive pasture-based feed system. J. Dairy Sci. 93:4302-4317. https://doi.org/10.3168/jds.2009-2591.

Craig, H. J. B., K. Stachowicz, M. Black, M. Parry, C. Burke, S. Meier, and P. Amer. 2018. Genotype by environment interactions in fertility traits in New Zealand dairy cows. J. Dairy Sci. 101:1099111003. https://doi.org/10.3168/jds.2017-14195.

Crump, R., N. Wray, R. Thompson, and G. Simm. 1997. Assigning pedigree beef performance records to contemporary groups taking account of within-herd calving patterns. Anim. Sci. 65:193-198. https://doi.org/10.1017/S1357729800016490.

Dezetter, C., H. Leclerc, S. Mattalia, A. Barbat, D. Boichard, and V. Ducrocq. 2015. Inbreeding and crossbreeding parameters for production and fertility traits in Holstein, Montbéliarde, and Nor- mande cows. J. Dairy Sci. 98:4904-4913. https://doi.org/10.3168/ jds.2014-8386.

Dunne, F. L., M. M. Kelleher, S. W. Walsh, and D. P. Berry. 2018. Characterization of best linear unbiased estimates generated from national genetic evaluations of reproductive performance, survival, and milk yield in dairy cows. J. Dairy Sci. 101:7625-7637. https:/ /doi.org/10.3168/jds.2018-14529.

Ferreira, J. L., A. Souza de Assis, F. Brito Lopes, T. Wayne Murphy, M. Corrêa da Silva, J. A. Soares Garcia, and E. G. Marques. 2015. Reaction norms in weights at 365 days old in Nellore bulls in northern Brazil. Semin. Cienc. Agrar. 36:4613-4626. https://doi .org/10.5433/1679-0359.2015v36n6Supl2p4613.

Fikse, W. F., R. Rekaya, and K. A. Weigel. 2003. Assessment of environmental descriptors for studying genotype by environment interaction. Livest. Prod. Sci. 82:223-231. https://doi.org/10.1016/ S0301-6226(03)00009-5.

Gilmour, A. R., B. Gogel, B. Cullis, R. Thompson, and D. Butler. 2009. ASReml user guide release 3.0. VSN Int. Ltd., Hemel Hempstead, UK.

Haile-Mariam, M., M. Carrick, and M. Goddard. 2008. Genotype by environment interaction for fertility, survival, and milk production traits in Australian dairy cattle. J. Dairy Sci. 91:4840-4853. https: //doi.org/10.3168/jds.2008-1084.

Irish Cattle Breeding Federation. 2017. Update to economic values in the EBI. Accessed Nov. 20, 2018. www.icbf.com/wp/?p=9695.

Kargo, M., P. Madsen, and E. Norberg. 2012. Is crossbreeding only beneficial in herds with low management level? J. Dairy Sci. 95:925-928. https://doi.org/10.3168/jds.2011-4707.

Kelleher, M., F. Buckley, R. Evans, and D. P. Berry. 2016. Additive genetic, non-additive genetic and permanent environmental effects for female reproductive performance in seasonal calving dairy females. Ir. J. Agric. Food Res. 55:10-23.

McAllister, A. 2002. Is crossbreeding the answer to questions of dairy breed utilization? J. Dairy Sci. 85:2352-2357. https://doi.org/10 .3168/jds.S0022-0302(02)74315-4.

MiX99 Development Team. 2015. MiX99: A software package for solving large mixed model equations. Natural Resources Institute Finland (Luke), Jokioinen, Finland.

Mulder, H., R. Veerkamp, B. Ducro, J. Van Arendonk, and P. Bijma. 2006. Optimization of dairy cattle breeding programs for different environments with genotype by environment interaction. J. Dairy Sci. 89:1740-1752. https://doi.org/10.3168/jds.S0022 -0302(06)72242-1.

Penasa, M., M. De Marchi, R. Dal Zotto, G. De Jong, G. Bittante, and M. Cassandro. 2010. Heterosis effects in a black and white dairy cattle population under different production environments. Livest. Sci. 131:52-57. https://doi.org/10.1016/j.livsci.2010.02.027.

Pollott, G. E., and J. Greeff. 2004. Genotype $\times$ environment interactions and genetic parameters for fecal egg count and production traits of Merino sheep. J. Anim. Sci. 82:2840-2851. https://doi .org $/ 10.2527 / 2004.82102840 x$.

Schmitz, F., R. W. Everett, and R. L. Quaas. 1991. Herd-year-season clustering. J. Dairy Sci. 74:629-636. https://doi.org/10.3168/jds .S0022-0302(91)78210-6.

Simm, G. 1998. Genetic Improvement of Cattle and Sheep. Farming Press, Tonbridge, UK.

Sørensen, M. K., E. Norberg, J. Pedersen, and L. Christensen. 2008. Invited review: Crossbreeding in dairy cattle: A Danish perspective. J. Dairy Sci. 91:4116-4128. https://doi.org/10.3168/jds.2008 -1273 .

Visscher, P. M., W. G. Hill, and N. R. Wray. 2008. Heritability in the genomics era-Concepts and misconceptions. Nat. Rev. Genet. 9:255-266.

Wall, E., S. Brotherstone, J. Kearney, J. Woolliams, and M. Coffey. 2005. Impact of nonadditive genetic effects in the estimation of breeding values for fertility and correlated traits. J. Dairy Sci. 88:376-385. https://doi.org/10.3168/jds.S0022-0302(05)72697-7. 